\title{
Epidemiological Modeling of Measles Infection with Optimal Control of Vaccination and Supportive Treatment
}

\author{
Okey Oseloka Onyejekwe, Esayas Zewdie Kebede \\ Computational Science Program, Addis Ababa University, Arat Kilo Campus, Addis Ababa Ethiopia
}

Email address:

okuzaks@yahoo.com (O. O. Onyejekwe) esachewzew@gmail.com (E. Z. Kebede)

\section{To cite this article:}

Okey Oseloka Onyejekwe, Esayas Zewdie Kebede. Epidemiological Modeling of Measles Infection with Optimal Control of Vaccination and Supportive Treatment. Applied and Computational Mathematics. Vol. 4, No. 4, 2015, pp. 264-274. doi: 10.11648/j.acm.20150404.15

\begin{abstract}
We consider an SEIR model with constant population size and formulate an optimal control problem subject to vaccination and supportive treatment as controls. Our aim is to find the optimal combination of vaccination and supportive treatment strategies that will minimize the cost of the two control measures as well as the number of infectives while efficiently balancing vaccination and management of measles applied to the models with various cost scenarios. We used Pontryagin's maximum principle to characterize the optimal levels of the two controls. The resulting optimality system is solved numerically by forward-backward sweep method. The results show that the optimal combination of the strategies required to achieve the set objective will depend on the relative cost of each of the control measures and the resulting optimality system showed that, the use of vaccinating and supportive treating at the same time at the highest possible rate to the population as early as possible is essential for controlling measles epidemic. The results from our simulation are discussed.
\end{abstract}

Keywords: Measles, Optimal Control, Pontryagin's Maximum Principle, Adjoint Condition, Transversality Condition, Hamiltonian, Optimality System

\section{Introduction}

Most infectious diseases could be driven towards eradication, if adequate and timely steps (e.g. vaccination, treatment, educational and enlightenment campaign, etc.) are taken in the course of the epidemic [7, 10, 11, 12]. However, many of these diseases eventually become endemic in many societies due to lack of adequate policies and timely interventions to mitigate the spread of the diseases [14]. Consequently, there is the need for proactive steps towards controlling the spread of infectious diseases, particularly those ones for which both vaccine and cure are available. Moreover, it is often cheaper to prevent the occurrence of a disease with management of treatment strategies than to cure it.

Measles is an acute, highly communicable, viral infectious human disease cause serious illness, life-long complications and death caused by measles virus (paramyxovirus) [1, 2, 3, 4, 5]. It is still a public health problem in many developing countries. According to the World Health Organization (WHO) $[1,2,3]$, more than 20 million people are affected by measles each year with more than $95 \%$ of measles deaths occur in countries that have low per capita incomes and weak health infrastructures particulary in Africa and Asia. Measles epidemics often occur every two to three years and usually last between two and three months [2, 3, 4]. In the year 2012, the World Health Organization (WHO) estimated that 122,000 children died of measles. The majority of these occur in developing countries with more than half in sub-Saharan Africa. This disease burden accounts for $15 \%$ of all under-five mortality $[1,2,3,4,6]$.

Measles can be transmitted by direct contact with infected nasal or throat secretions or transmission through coughing and sneezing of infected people. Humans are the only natural hosts of measles virus. As a result, it can spread rapidly by contact in a susceptible population $[2,3]$.

Clinical features of measles can be divided into four stages of illness period the first stage is incubation period, this stage is approximately 10-12 days from exposure to the onset of fever and other nonspecific symptoms and average 14 days. The second, prodrome period, during this period, symptoms appear. They usually begin 12-14 days after exposure. Common symptoms include: fever, fatigue, decreased appetite, red watery eyes, runny nose, cough and often peaking as high as $38^{\circ} \mathrm{C}$. The third stage is exanthem (rash) period, during this period, a rash develops. The rash usually starts on the face and spreads to the neck, trunk, arms and legs. The last stage is recovery period, a cough may last for 1-2 weeks after the 
measles infection $[2,13]$. There is no specific medicine that kills the measles virus. The best way to reduce the risk of contracting measles is to be immunized with the measles vaccine. In the absence of vaccination, the measles virus would infect almost 100 percent of the population [1, 2, 3, 4,].

Optimal control theory is an area of mathematics that is used extensively in controlling the spread of infectious diseases. It is a powerful mathematical tool in the late 1950s, and has been used successfully to make decisions involving complex biological situations. It is often used in the control of the spread of most diseases for which either vaccine or treatment is available $[8,15,16,17,18,25,26]$.

In this paper we intended to answer the question of how to optimally combine the vaccination and the supportive treatment schedules and control strategies such that the cost of the implementation of the two interventions is minimized while the disease is eradicated within a specified period.

\section{Model Formulation}

In this paper, we formulate an SEIR (susceptible, exposed, infected and recovered) model for measles with the optimal control strategies. In a typical measles infection scenario, when mothers have been infected, immunoglobulin ( $\mathrm{IgG}$ ) antibodies transferred across the placenta to newborn infants give them temporary passive immunity to measles infection. After that, maternal antibodies remain in the body up to nine months. It can therefore be assumed that an infant enters directly into the susceptible class $\mathrm{S}$ at birth. When there is an adequate contact of a susceptible with an infective disease transmission occurs, and the susceptible enters the exposed class $\mathrm{E}$ of those in the latent period, who are infected but not yet infectious. At the end of the latent period, an individual enters the class I of infectives. These can be considered infectious in the sense that they are capable of transmitting the infection. When the infectious period ends, the individual enters the recovered class $\mathrm{R}$ consisting of those with permanent infection-acquired immunity otherwise they pass away. We exclude vertical incidence in our model, this obviates the infection rate of newborns by their mothers. Our model belong SEIR transmission model $[18,40]$.

\section{Model Assumptions}

1. The population is uniform and mixes homogeneously. The total population size, $\mathrm{N}$, is constant, i.e.
$N(t)=S(t)+E(t)+I(t)+R(t)$ at any time $t \geq 0$.

2. The natural birth $b$ and death rates $\mu$ are assumed to be equal. With rapid urbanization and economic diversification, this assumption can be considered valid in many developing countries.

3. The infectious $I$ move from their compartment to R-compartment at a constant rate $\gamma$, and latent's $\mathrm{E}$ move from their compartment to $I$ - compartment at a constant rate $\varepsilon$, so that $1 / \gamma$ is the mean infectious period and $1 / \mathcal{E}$ is the mean latent period;

4. Each individual in the population is considered as having an equal probability of contacting the disease with a contact rate $\beta$;

5. An infected individual makes contact and is able to transmit the disease with $\beta N$ others per unit time, that is, the contact rate is proportional to the total population size.

6. The fraction of contacts by an infected with a susceptible is $S / N$. Therefore the number of new infections in unit time per infective becomes $(\beta N)(S / N)$. This rate is called an infection rate. This gives the rate of new infections or those leaving the susceptible category as $(\beta N)(S / N) I=\beta S I$, which is called an incidence of the disease.

7. The number of infected individuals from the exposed compartment per unit time is $\varepsilon E$ at time $\mathrm{t}$ and the number of recovered individuals from the infected compartment per unit time is $\gamma I$ at time $\mathrm{t}$.

8. The rate of susceptible, exposed, infected \& recovered individual removed from each compartments through natural death is $\mu S, \mu E, \mu I$, and $\mu R$ respectively.

9. Individuals in the recovered class are assumed to be immune for life;

\section{Model with Controls}

In this section, an optimal control problem is formulated by incorporating two intervention strategies.

(i) $u(t)$ is the control which represents the percentage of susceptible individuals being vaccinated per unit of time.

(ii) $v(t)$ is the control effort aimed at treating infected individuals being supportively treated per unit of time.

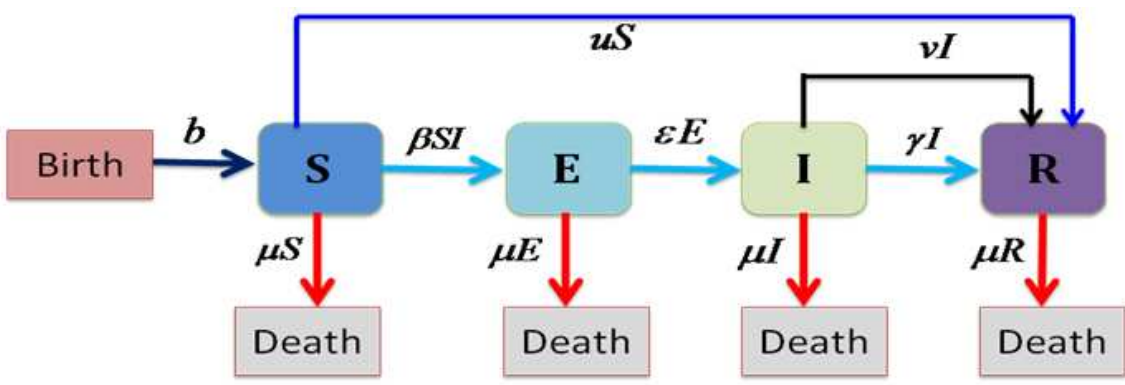

Figure 1. The SEIR schematic model with control $u$ and $v$ 
The vaccine and supportive treatment drives the susceptible and infected individuals to the recovered class, respectively. So we introduce the control function $u(t)$ and $v(t)$ and in order to set up an optimal control problem related to the SEIR epidemic model.

Table 1.1. Summary of description of variables and parameters for the measles model.

\begin{tabular}{ll}
\hline Symbols & Description \\
\hline$S$ & Susceptibles population \\
$E$ & Exposed population \\
$I$ & Infectives population \\
$R$ & Recovered population \\
$\beta$ & Transmission Rate \\
$\mu$ & Average Death Rate \\
$b$ & Average Birth Rate \\
$\mathcal{E}$ & Latency Rate \\
$\gamma$ & Recovery Rate \\
$1 / \varepsilon$ & Average Latent period \\
$1 / \gamma$ & Average Infectious Period \\
\hline
\end{tabular}

Based on the assumption and diagram we drive the following differential equations (state equations) of the dynamics measles transmission model is given in equation (1).

$$
\left\{\begin{array}{l}
\frac{d S}{d t}=b-\beta S I-\mu S-u S \\
\frac{d E}{d t}=\beta S I-(\varepsilon+\mu) E \\
\frac{d I}{d t}=\varepsilon E-(\gamma+\mu+v) I \\
\frac{d R}{d t}=\gamma I-\mu R+u S+v I
\end{array}\right.
$$

With initial conditions

$$
\left\{\begin{array}{l}
S(0)=S_{0} \geq 0, \\
E(0)=E_{0} \geq 0, \\
I(0)=I_{0} \geq 0, \\
R(0)=R_{0} \geq 0
\end{array}\right.
$$

\section{Optimal Control Problem}

A typical optimal control problem requires a cost functional $(J[x(t), u(t), v(t)])$, a set of state variables $(x(t) \in X)$ and a set of control variables $(u(t) \in U)$ in a time $t, 0 \leq t \leq T$.

We aim to minimize the objective (cost) functional $J$ considering the costs of vaccination of susceptible human and costs with supportive treatment of infected human given by:

$$
J(u, v)=\int_{0}^{T}\left(A_{1} E+A_{2} I+\frac{1}{2} B_{1} u^{2}+\frac{1}{2} B_{2} v^{2}\right) d t
$$

subject to the differential equations (1) where:

- The control set $U$ is measurable functions and it is defined as:

$$
U=\left\{(u(t), v(t)) \mid 0 \leq u \leq u_{\max }<1,0 \leq v \leq v_{\max }<1, t \in[0, T]\right\}
$$

- $u_{\max }$ is maximum attainable value for $u$ and $v_{\max }$ is maximum attainable value for $v$ because resources are limited.

- $B_{1}$ and $B_{2}$ are positive constants representing the relative weights attached to the cost of vaccination and cost of supportive treatment respectively.

- $A_{1}$ and $A_{2}$ are constants to keep a balance in the size of $\mathrm{E}$ and $I$ in the functional objective, respectively.

- $[0, \mathrm{~T}]$ represents the control period $[0,90]$ in days.

The second term in the functional objective (as it is customary) the quadratic term (cost) $\frac{1}{2} B_{1} u^{2}$ and $\frac{1}{2} B_{2} v^{2}$ take a nonlinear function, where $B_{1}$ and $B_{2}$ are positive weight parameters associated with the control $u$ and $v$. Weight parameter (B) 'measures' the comparative importance of reducing the disease burden compared to reducing the vaccination and treatment costs.

The control $u$ is the percentage of the susceptible that is vaccinated per unit time while the control $v$ is the percentage of the infectives that are supportively treated per unit time. Thus, $u$ and $v$ lie between 0 and 1 while $u_{\max }$ and $v_{\max }$ will depend on the amount of resources available to implement each of the control measures. If $u=0, v=0$ then no vaccination and no treatment is done hence the extended model (1) is considered as uncontrolled. When $u=1, v=1$ indicate that all susceptible and infectious population are vaccinated and treated respectively. The rate of vaccination and treatment are assumed to take values in the range of [0, 0.9] instead of $[0,1]$ respectively in order to eliminate the case where the entire susceptible and infectious population are vaccinated and treated $[15,20,22,26,27]$.

The weights $B_{1}$ and $B_{2}$ will depend on the relative importance of each of the control measures in mitigating the spread of the disease as well as the cost (human effort, material resources, infrastructural resources, etc.) of implementing each of the control measures per unit time. The vaccination cost could include the cost of the vaccine, cost of syringes, cost of safety boxes, the vaccine storage cost, other related overheads, etc. The supportive treatment cost should include the cost of the medical tests and diagnosis, drug cost, hospitalization cost if needed etc [22].

Our target is to minimize the objective functional defined above by decreasing the number of infected and exposed individuals and increasing the number of recovered individuals by using possible minimal control variables $u$ and $v$ (or minimize the cost of the vaccination and the cost of the supportive treatment at each time unit within the implementation period).

The Optimal Control Problem of nonlinear dynamics of the SEIR measles epidemic model given by; 
$\min _{u, v} J(u, v)=\min \int_{0}^{T}\left(A_{1} E+A_{2} I+\frac{1}{2} B_{1} u^{2}+\frac{1}{2} B_{2} v^{2}\right) d t$

Subject to :

control set defined as;

$$
\left\{\begin{array}{l}
\frac{d S}{d t}=b-\beta S I-\mu S-u S \\
\frac{d E}{d t}=\beta S I-(\varepsilon+\mu) E \\
\frac{d I}{d t}=\varepsilon E-(\gamma+\mu) I-v I \\
\frac{d R}{d t}=\gamma I-\mu R+u S+v I
\end{array}\right.
$$

$$
U=\left\{(u(t), v(t)) \mid 0 \leq u \leq u_{\max }<1,0 \leq v \leq v_{\max }<1, t \in[0, T]\right\}
$$

\section{Pontryagin's Maximum Principle-PMP}

We consider controls $u$ and $v$, together with state variables $\mathrm{S}, \mathrm{E}, \mathrm{I}$ and $\mathrm{R}$ including respective adjoint functions $\lambda_{1}, \lambda_{2}, \lambda_{3}$ and $\lambda_{4}$ The Hamiltonian function is given by

$$
\left\{\begin{aligned}
H\left(t, S, E, I, R, u, v, \lambda_{1}, \lambda_{2}, \lambda_{3}, \lambda_{4}\right)= & f(t, S, E, I, R, u, v)+\lambda_{i} \sum_{i=1}^{4} g_{i}(t, S, E, I, R, u, v) \\
& \Rightarrow f(t, S, E, I, R, u, v)+\lambda_{1} S^{\prime}+\lambda_{2} E^{\prime}+\lambda_{3} I^{\prime}+\lambda_{4} R^{\prime}
\end{aligned}\right.
$$

where $S, E,,^{\prime} I, R^{\prime}$ are obtained from (1)

$$
H=\left(A_{1} E+A_{2} I+\frac{1}{2} B_{1} u^{2}+\frac{1}{2} B_{2} v^{2}\right)+\left[\begin{array}{c}
\lambda_{1}(\mu-\beta S I-\mu S-u S)+ \\
\lambda_{2}(\beta S I-(\varepsilon+\mu) E)+ \\
\lambda_{3}(\varepsilon E-(\gamma+\mu) I-v I)+ \\
\lambda_{4}(\gamma I-\mu R+u S+v I)
\end{array}\right]
$$

Next, by applying Pontryagin's maximum principle to the Hamiltonian, we obtain the following results.

Given optimal control $\left(u^{*}, v^{*}\right)$ pair and the corresponding solution $S^{*}, E^{*}, I^{*}$ and $R^{*}$ of systems (4), there exist adjoint variables $\lambda_{1}, \lambda_{2}, \lambda_{3}$, and $\lambda_{4}$ that satisfy the adjoint condition $[15,31,37,38,5051]$;

$$
\lambda_{i}^{\prime}(t)=-\frac{\partial H}{\partial x_{i}} \Rightarrow \lambda_{i}^{\prime}=-\left(f+\lambda_{i} \sum_{i=1}^{4} g_{i}\right) x_{i}
$$

Here $x_{i}, i=1,2,3,4$ are the state variables $S, E, I$ and $R$, and $g_{i}$ are the right hand sides of the system (1). We find the adjoint function for each state variable S,E, $I$ and $\mathrm{R}$ for system of equation (4)

The adjoint equations are:

$$
\left\{\begin{array}{l}
\lambda_{1}^{\prime}=\lambda_{1}(\beta I+\mu+u)-\lambda_{2}(\beta I)-\lambda_{4}(u) \\
\lambda_{2}^{\prime}=-A_{1}+\lambda_{2}(\varepsilon+\mu)-\lambda_{3}(\varepsilon) \\
\lambda_{3}^{\prime}=-A_{2}+\lambda_{1}(\beta S)-\lambda_{2}(\beta S)+\lambda_{3}((\gamma+\mu)+v)-\lambda_{4}(\gamma+v) \\
\lambda_{4}^{\prime}=\lambda_{4} \mu
\end{array}\right.
$$

with transversality conditions in (9)

The state variables are not assigned at the final time $T$ so that we have the transversality equations:

$$
\lambda_{i}\left(t_{f}\right)=0, i=1,2,3,4
$$

In order to illustrate the characterization of the optimal control $u^{*}$ and $v^{*}$, we consider first the optimality condition.

The optimality condition for each control $u^{*}$ and $v^{*}$ from Hamiltonian in (6) yields

$$
\begin{aligned}
\frac{\partial H}{\partial u}=0 \text { at } u=u^{*} & \Rightarrow f_{u}(t, x, u)+\lambda g_{u}(t, x, u)=0 \\
\Rightarrow & B_{1} u+\lambda_{1}(-S)+\lambda_{4}(S), \text { at } u=u^{*} \\
& u^{*}=\left(\lambda_{1}-\lambda_{4}\right) \frac{S}{B_{1}} \\
\frac{\partial H}{\partial v}=0 \text { at } v=v^{*} \Rightarrow & f_{v}(t, x, u)+\lambda g_{v}(t, x, u)=0 \\
\Rightarrow & B_{2} v+\lambda_{3}(-I)+\lambda_{4}(I), \text { at } v=v^{*} \\
& v^{*}=\left(\lambda_{3}-\lambda_{4}\right) \frac{I}{B_{2}}
\end{aligned}
$$


taking into account the bounds on $u^{*}$ and $v^{*}$ the

$$
u^{*}=\left\{\begin{array}{ll}
0 & \text { if } \frac{\partial H}{\partial u}>0 \\
\left(\lambda_{1}-\lambda_{4}\right) \frac{S}{B_{1}} & \text { if } \frac{\partial H}{\partial u}=0 \\
0.9 & \text { if } \frac{\partial H}{\partial u}<0
\end{array} \quad \text { and } \quad v^{*}= \begin{cases}0 & \text { if } \frac{\partial H}{\partial v}>0 \\
\left(\lambda_{3}-\lambda_{4}\right) \frac{I}{B_{2}} & \text { if } \frac{\partial H}{\partial v}=0 \\
0.9 & \text { if } \frac{\partial H}{\partial v}<0\end{cases}\right.
$$

so the optimal control pair $\left(u^{*}, v^{*}\right)$ may be written in short form:

$$
\mid \begin{aligned}
& u^{*}=\max \left(\min \left(\left(\lambda_{1}-\lambda_{4}\right) \frac{S}{B_{1}}, 0.9\right), 0\right) \\
& v^{*}=\max \left(\min \left(\left(\lambda_{3}-\lambda_{4}\right) \frac{I}{B_{2}}, 0.9\right), 0\right)
\end{aligned}
$$

The optimal control pair and the state are found by solving the following optimality system, which consists of the state system (1), the adjoint system (8), tranversality conditions (9) and the characterization of the optimal control pair $\left(u^{*}, v^{*}\right)$ (13). The following system which characterizes the optimal control is called the optimality system.

$$
\left\{\begin{array}{l}
S^{\prime}=b-\beta S I-\mu S-u^{*} S \\
E^{\prime}=\beta S I-(\varepsilon+\mu) E \\
I^{\prime}=\varepsilon E-\left(\gamma+\mu+v^{*}\right) I \\
R^{\prime}=\gamma I-\mu R+u^{*} S+v^{*} I \\
S(0)=S_{0} \geq 0, E(0)=E_{0} \geq 0, I(0)=I_{0} \geq 0, R(0)=R_{0} \geq 0 \\
\lambda_{1}^{\prime}=\lambda_{1}\left(\beta I+\mu+u^{*}\right)-\lambda_{2}(\beta I)-\lambda_{4} u^{*} \\
\lambda_{2}^{\prime}=-A_{1}+\lambda_{2}(\varepsilon+\mu)-\lambda_{3}(\varepsilon) \\
\lambda_{3}^{\prime}=-A_{2}+\lambda_{1}(\beta S)-\lambda_{2}(\beta S)+\lambda_{3}\left((\gamma+\mu)+v^{*}\right)-\lambda_{4}\left(\gamma+v^{*}\right) \\
\lambda_{4}^{\prime}=\lambda_{4} \mu \\
\lambda_{i}\left(t_{f}\right)=0, \quad i=1,2,3,4
\end{array}\right.
$$

\section{Simulation Results and Discussion}

In this section, we solve numerically the optimality system (14) using the forward-backward sweep method developed by Suzanne lenhart and J.T.Workman [31]. The numerical procedure begins with an initial guess on the control variable; then, the state equations are solved simultaneously forward in time, followed by the adjoint equations which are simultaneously solved backward in time. The control is updated by inserting the new values of states and adjoints into its characterization, and the process is repeated until convergence occurs. The ODE solver used for the state and adjoint systems is a Runge-Kutta fourth order procedure executing using MATLAB $[8,15]$.

Table 1.2. Epidemiological parameters used for the numerical simulations.

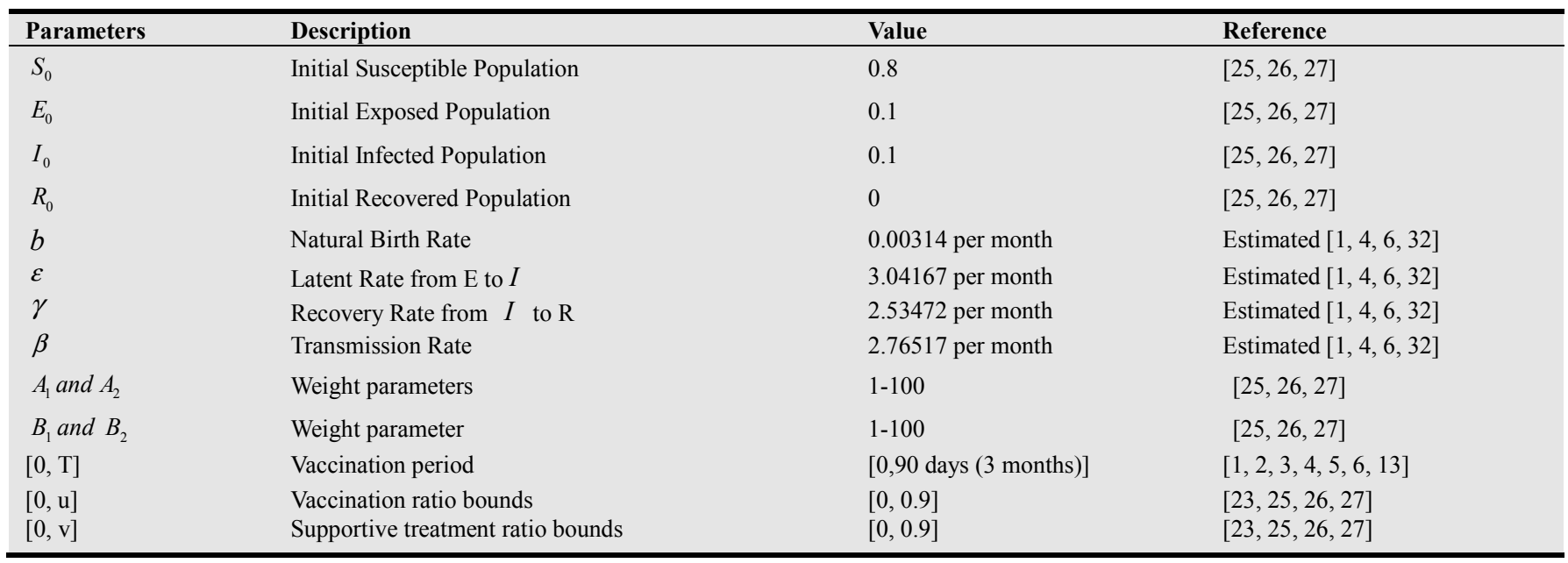




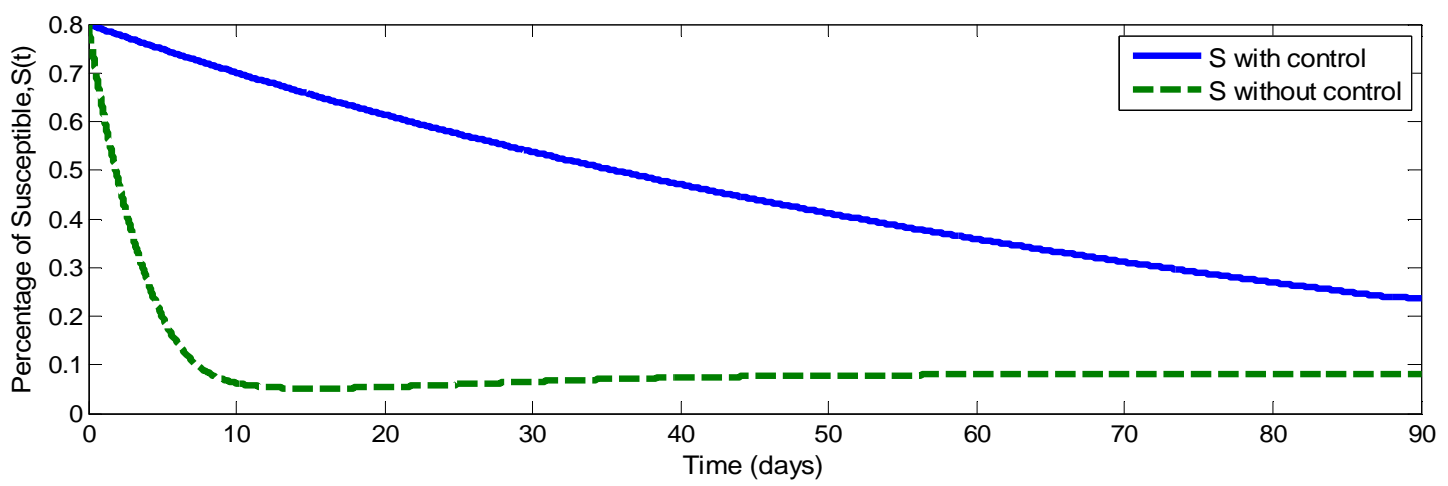

Figure 2a. Comparison of susceptible individuals under an optimal control situation and no control (for $A_{1}=100, A_{2}=100, B_{1}=1, B_{2}=1$ ).

In figure $2 \mathrm{a}$, we show the numerical solution of controlled and uncontrolled susceptible populations. There is a significant decrease in the number of susceptible with control compared with those not vaccinated. As vaccination is not given to the susceptible individual, during the first 12 day latent period, large amount of susceptible individuals can be exposed to the disease and steadily decreases until it starts to slowly increase in the last instants of the interval. On the contrary, vaccinated susceptible group develops an immunity which keeps it from moving to the exposed class. Even if they are in contact with infected individual the vaccine protects them from measles infection.

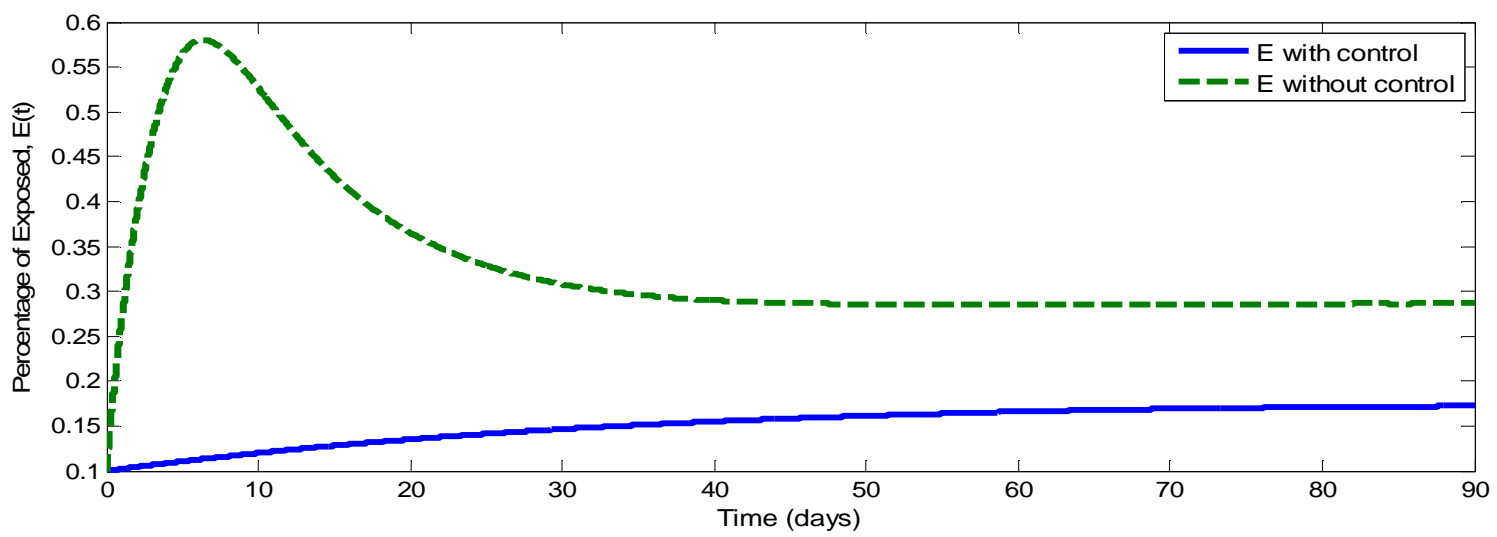

Figure 2b. Comparison of exposed individuals under an optimal control situation and no control (for $A_{1}=100, A_{2}=100, A_{3}=100, B_{1}=1, B_{2}=1$ ).

In figure $2 b$, we show the numerical solution of controlled and uncontrolled exposed populations. Since in figure $2 \mathrm{a}$ unvaccinated susceptible individuals move fast to exposed class during the latent period (on the first 12 days), because of this, figure $2 b$ shows that the exposed individuals increased during latent period which peaks at about $0.57 \%$ population. However, in case of the uncontrolled class, a small number of individuals move to the exposed class. This results in reducing the exposed individuals from the measles disease. After 60 days both groups approach stable states.

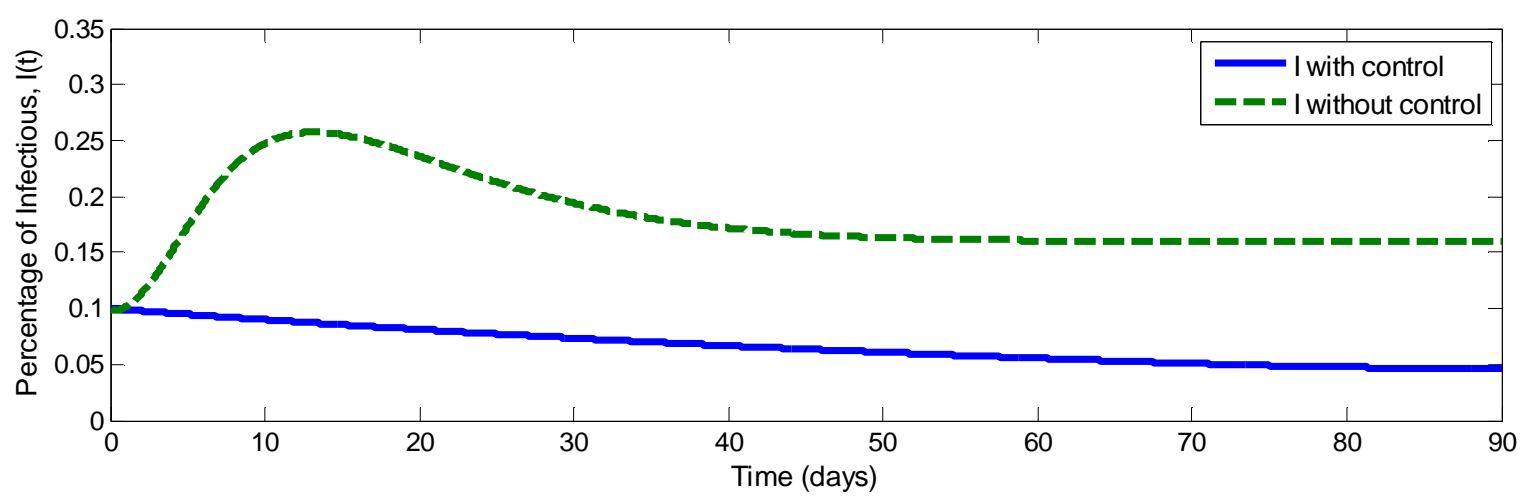

Figure 2c. Comparison of infected individuals under an optimal control situation and no control (for $A_{1}=100, A_{2}=100, B_{1}=1, B_{2}=1$ ).

Fig. 2c, illustrates that control of infected individuals results in reducing the infected individuals (to $0.05 \%$ ) of population.
This is because supportive treatment is given to this group. It should be noted that large numbers of exposed individual 
move to this class during the latent period and after latent

period the number decreases because of the recovery rate.

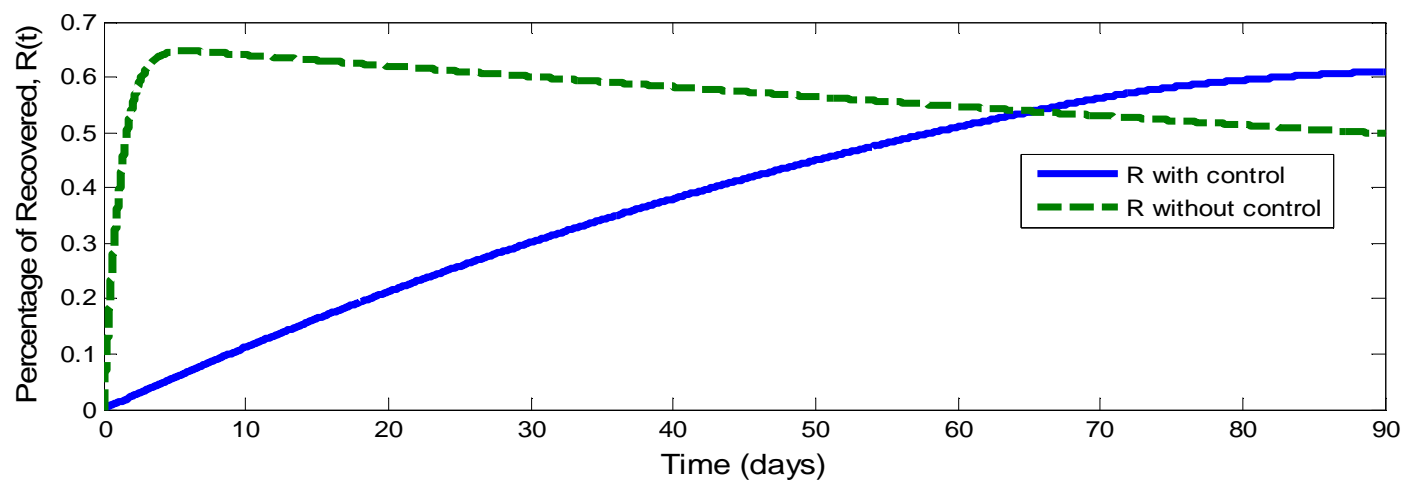

Figure 2d. Comparison of recovered individuals under an optimal control situation and no control (for $A_{1}=100, A_{2}=100, B_{1}=1, B_{2}=1$ ).

Figure 2d, demonstrates the dynamics of the recovered class with and without control measures. The recovered class without any control increases faster than control during the control period. This is because as large numbers of individuals enter into this group from the susceptible, exposed, and infected class. The recovered classes with control increases continuously until the populations developed immunity.

Generally it can be seen from Figs. 2a-2d that the optimal vaccination and supportive treatment policies have a profound

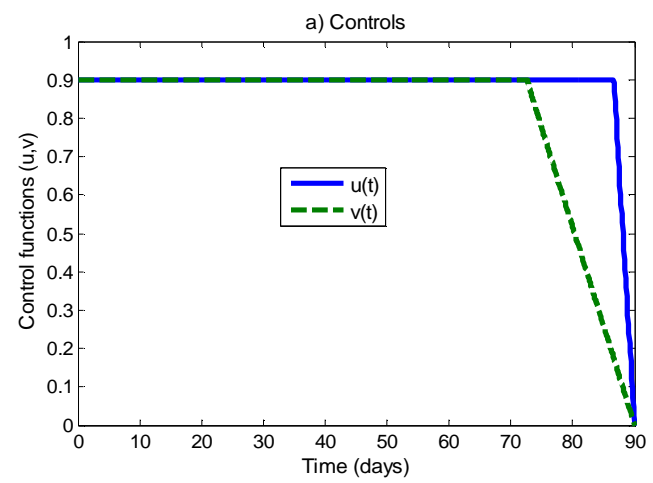

effect on the spread and control of measles epidemic. In addition, the overall dynamics clearly demonstrates the impact of intervention before 12 days of the epidemic is more substantial. We also observe that when the population is uncontrolled (no vaccination and no supportive treatment) almost all susceptible population is exposed and infected with the disease. The number of people in the susceptible, exposed and infected with control measures decreased more than without any control.

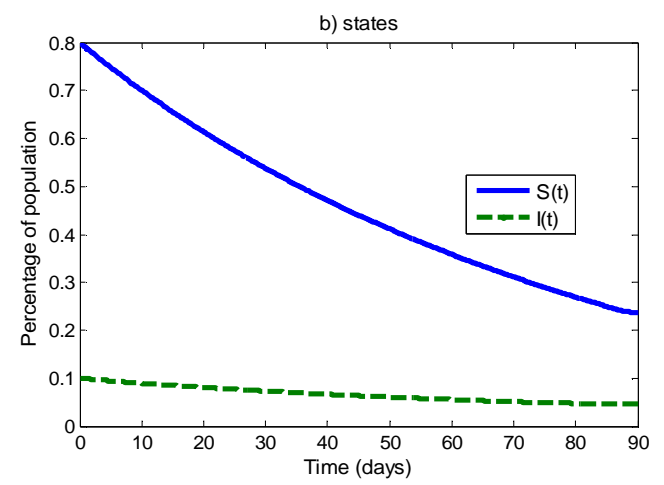

Figure 3 a and Fig. 3b. The control functions $u$ and $v$ a) on the left and susceptible and infectious individuals b) on the right versus time (for $A_{1}=100, A_{2}=100$, $B_{1}=1$ and $B_{2}=1$ ).

Fig. 3a illustrates the profile of the control functions $u$ and $v$ with weight $B_{1}=1, B_{2}=1$, the simulation shows the effects of vaccination and supportive treatment given for a much longer time. This results leads in a significant reduction of

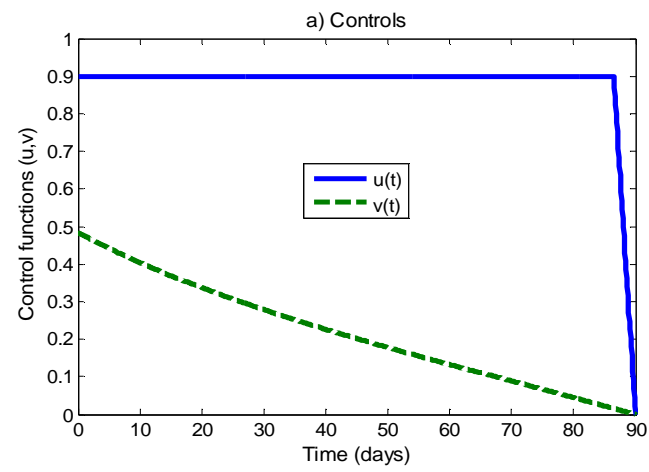

susceptible and the infected individuals over time. Figs. 3a and $3 \mathrm{~b}$ show that using both controls at the same time with the accompanying weights could minimize the number of susceptible and infected individuals significantly.

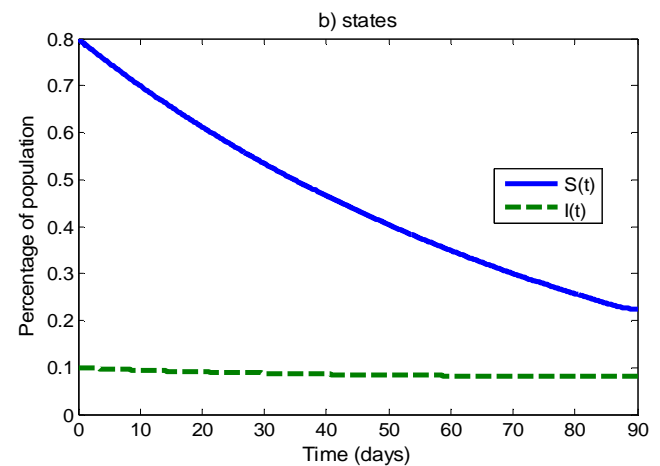

Figure $4 \boldsymbol{a}$ and $4 \boldsymbol{b}$. The control functions $u$ and $v$ a) on the left and susceptible and infectious individuals b) on the right versus time (for $A_{1}=100, A_{2}=100$, $B_{1}=1$ and $B_{2}=20$ ). 
Unlike before, the weights are increased $B_{1}=1$ and $B_{2}=20$. The results of the simulation clearly show that increasing the weight values for treatment results in a slightly

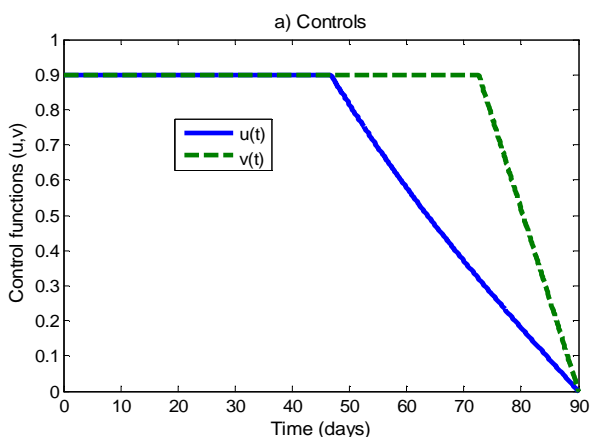

more increase in infected individuals. At the final time $(I=0.09 \%)$ as compared to the case for $B_{2}=1(I=0.05 \%)$.

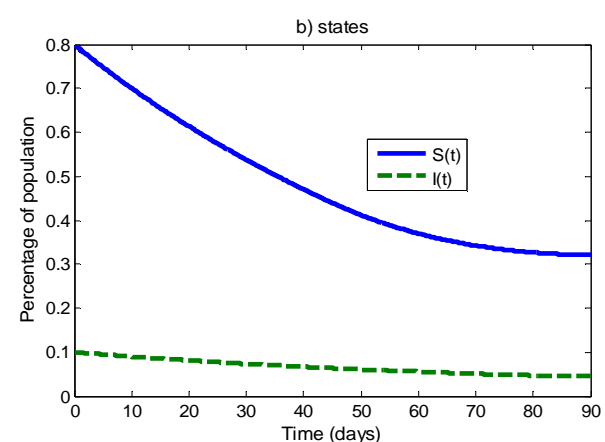

Figure 5a and 5b. The control functions $U$ and $v$ a) on the left and susceptible and infectious individuals $b$ ) on the right versus time (for $A_{1}=100, A_{2}=100$, $B_{1}=20$ and $B_{2}=1$ ).

Increasing the weight of vaccination $\left(B_{1}=20\right)$ results in higher susceptible individuals $(0.34 \%)$ when compared to $(0.22 \%)$ in $B_{1}=1$ case. So applying more of the vaccination

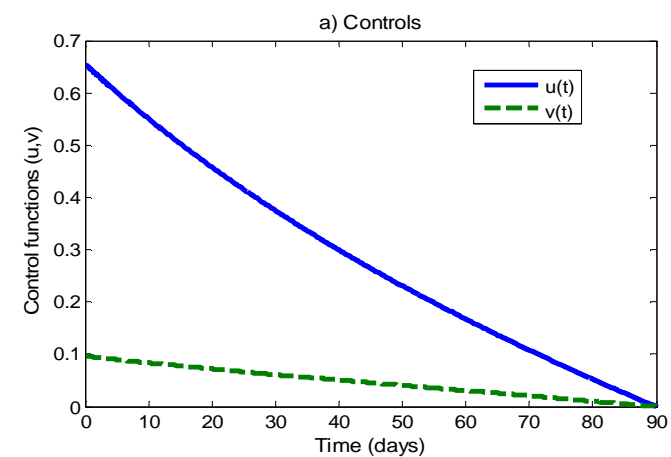

$B_{1}$ for a short period does not appreciably decreases the susceptible human population.

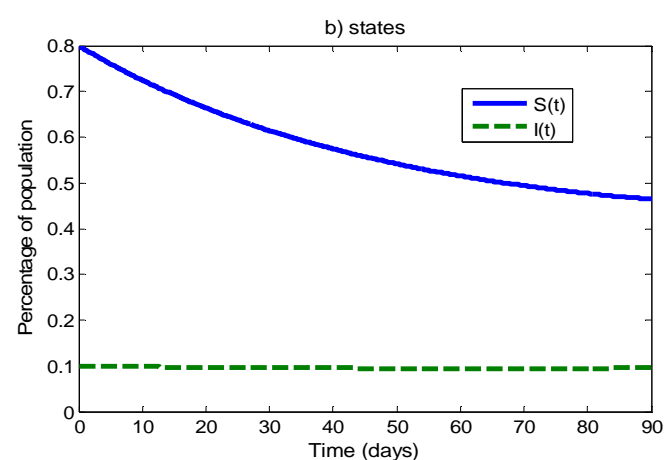

Figure $6 \boldsymbol{a}$ and $\mathbf{6} \boldsymbol{b}$. Control functions $u$ and $v$ a) on the left and susceptible and infectious individuals b) on the right versus time (for $A_{1}=100, A_{2}=100$, $B_{1}=100$ and $\left.B_{2}=100\right)$.

We demonstrate the effects of increments of weight values for both vaccination and supportive treatment $\left(B_{1}=100\right.$, $\left.\mathrm{B}_{2}=100\right)$. The above graphs show that the profiles of both control function are significantly altered. At the end of the control period, the susceptible and infectious individual show changes of $(\mathrm{S}=0.48, \mathrm{I}=0.1) \%$ in comparison with $(\mathrm{S}=0.22$, $\mathrm{I}=0.05) \%$. of the previous case. This implies that in order to mitigate the disease, initially we have to apply more of the supportive treatment control to reduce infection to a certain threshold, after which we can gradually start to apply more of the vaccination control with less of the treatment.

Figs 3-6 show that more care must be taken to control the disease burden at the beginning of the epidemic. This will involve a judicious application of vaccination and supportive treatment measures.

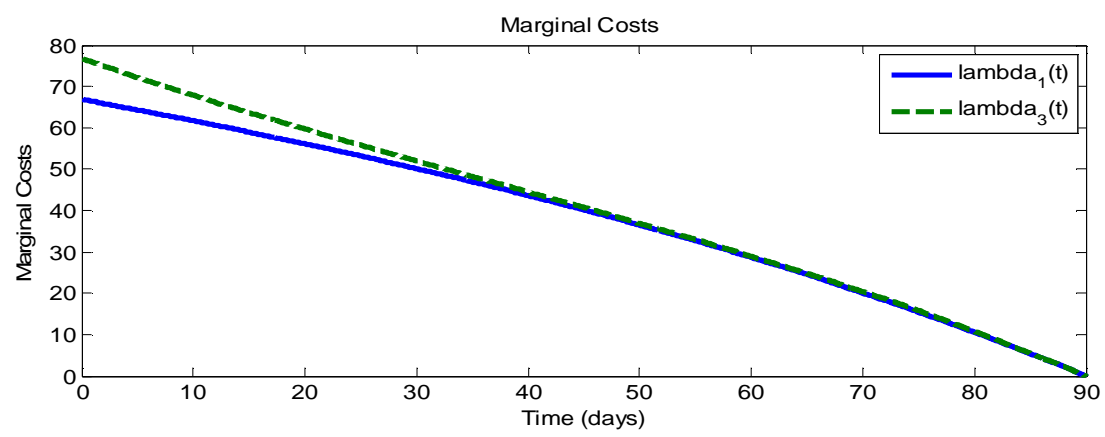

Figure 7. The marginal cost $\lambda_{1}$ and $\lambda_{3}$ (for $A_{1}=100, A_{2}=100, B_{1}=1$ and $\left.B_{2}=1\right)$. 
Figure 7 displays the numerical solutions for the adjoint variables for the case $B_{1}=1, B_{2}=1$.

The marginal cost of vaccination $\lambda_{1}$ is lower than marginal cost of supportive treatment $\lambda_{3}$ at the beginning of the control period. However for both controls, the cost is high initially and decreases continuously through the control period.

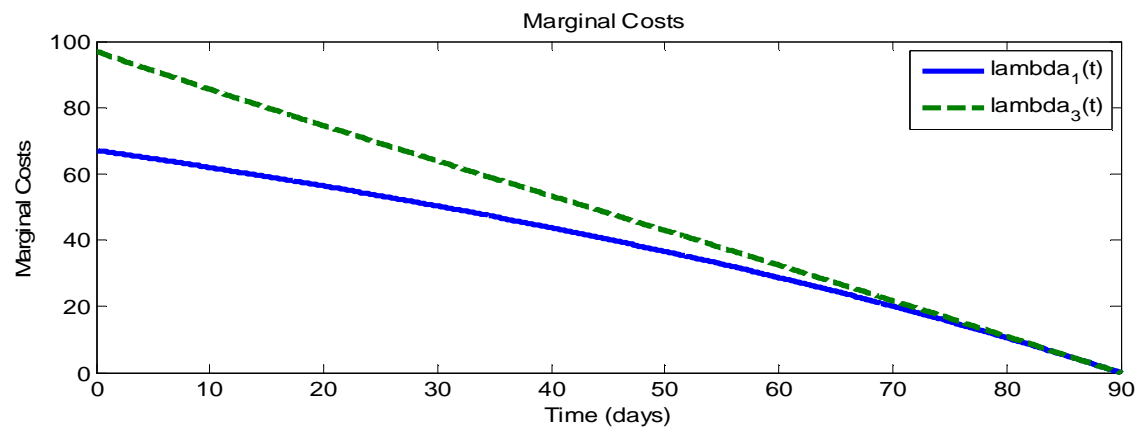

Figure 8. The marginal cost $\lambda_{1}$ and $\lambda_{3}$ (for $A 1=100, A 2=100, B_{1}=1$ and $B_{2}=20$ ).

For the given adjoint variables and for weights $B_{1}=1$, $B_{2}=20$ the prescribed weighted values of supportive treatment result in a decrease in the marginal cost of treatment.

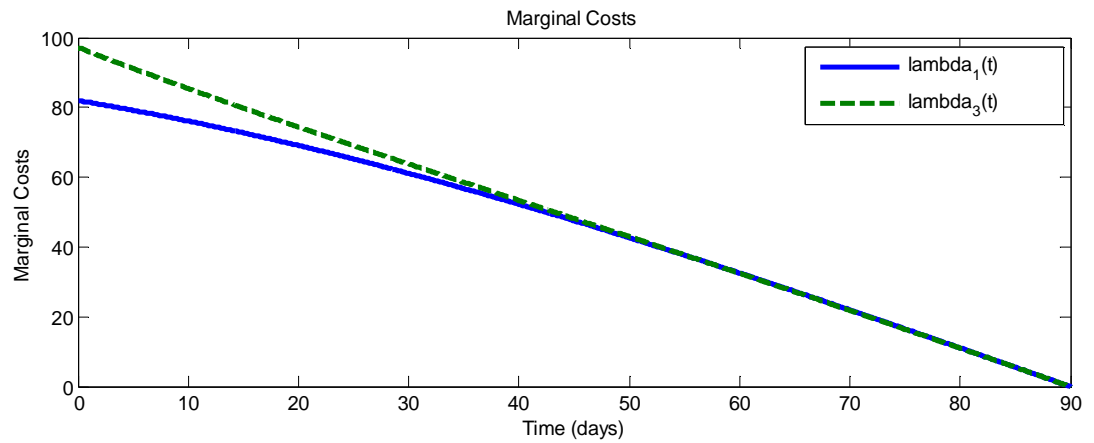

Figure 9. The marginal cost $\lambda_{1}$ and $\lambda_{3}$ (for $A_{1}=100, A_{2}=100, B_{1}=100$ and $\left.B_{2}=100\right)$.

Fig. 9 predicts an increase in marginal cost as the weight values of both controls are increased. However this does not result in a concomitant increase in outcome for both susceptible and infected individuals as was the case for relatively lower weights $\left(B_{1}=1\right.$ and $\left.B_{2}=1\right)$. This is because both the percentages of the population to be vaccinated and treated for the period are relatively small. This is still the case even if relatively higher weights are given (see Fig. 6b).

Figs. 7-9 show that the marginal cost of supportive treatment is higher than the marginal cost of vaccination in all cases in the first few weeks. Increasing the marginal cost of controls in the first few weeks cannot reduce both susceptible and infectious individuals, though the increase in the latter is more significant. This implies that it will be more economical to expand the vaccination coverage than to expand the supportive treatment coverage. However, after the overlap of the two costs, the marginal costs for both vaccination and supportive treatment begin to fall continually through the control period. Since, the marginal costs of the controls continue to decrease over time, this strategy is really advantageous to adopt especially in developing countries
However this decrease does not necessarily result in a decrease in infected individuals. (see Figs. 4a). where primary and supportive care for infectious diseases are often neglected.

\section{Conclusions}

The work presented herein demonstrates an optimal control strategy for measles disease subject to SEIR epidemiological model. We used Pontryagin's maximum principle to characterize the controls and derive an optimality system. A state of the art of uncontrolled and controlled mathematical models was employed to support the formulation.

The resulting optimality protocol displayed positive effects upon the population especially in the number of susceptible, exposed and infected individuals during the control period.

It was found that optimal control strategies become more effective in the control and containment of measles when vaccination and supportive treatment are combined optimally. This may require less weighting for longer control periods. The susceptible and infectious can be reduced up to $85 \%$ and $60 \%$ around the eighth week of the control period respectively. Since the cost of effectively managing measles decreases with 
time, a more proactive approach in combating the fight against measles is suggested.

The role played by the weight parameter B in the objective functional is made evident in this study. This parameter 'measures' the comparative importance of reducing the disease burden (see figures 3-9). In addition, it gives a clue concerning the relative importance of the application of treatment management strategies involving vaccination and supportive care in effectively controlling measles. It is shown that combined use of vaccination and supportive treatment is indicated as the most important intervention of any control strategy of measles epidemic.

Finally numerical simulations of the resulting optimality system showed that, in the case where it is more expensive to apply supportive treatment than vaccinate, resources should be invested in vaccination until the disease prevalence begins to fall. This option, however, will reduce the number of susceptible quickly enough, thus resulting in an overall decrease in the infected population. On the other hand, if it is more expensive to vaccinate than to supportively treat, then more resource must be put into care. Moreover, it is often cheaper to prevent the occurrence of a disease than to cure it.

\section{References}

[1] CDC, Progress in global measles control, Measles

[2] (http://www.cdc.gov/measles/pubs-mmwr.html)

[3] CDC, Morbidity and Mortality Weekly Report 2008-2015(MMWR) (https://surv.esr.cri.nz/surveillance/WeeklyMeaslesRpt.php)

[4] CDC, Global Measles Mortality, 2000-2008, MMWR

[5] (http://www.cdc.gov/mmwr/preview/mmwrhtml/mm5847a2.ht m)

[6] WHO/UNICEF, Strengthening Immunization Services through Measles Control, Joint Annual Measles Report 2010.

[7] WHO/UNICEF, Global Measles and Rubella Strategic Plan 2012- 2020 (2012).

[8] WHO/UNICEF, Measles and Rubella Initiative annual report, 2012.

[9] R.M. Anderson, R.M. May, Infectious Diseases of Humans Dynamics and Control, Oxford University Press, Oxford, 1991.

[10] Helena Sofia Ferreira Rodrigues, Optimal Control and Numerical Optimization Applied to Epidemiological Models, 2012.

[11] Herbert W. Hethcote, "The Mathematics of Infectious Diseases": SIAM Review, Vol. 42, No. 4. (December 2000).

[12] M.J. Keeling, B.T. Grenfell, Disease extinction and community size: modeling the persistence of measles, Science 275 (1997)

[13] M.J. Keeling, P. Rohani, Modeling Infectious Diseases in Humans and Animals, Princeton University Press, Princeton, NJ, 2007.
[14] M.J.Keeling and P.Rohani. Modeling infectious diseases in humans and animals. Princeton, NJ: Princeton University Press. xiii, 2008

[15] Ethiopian health and nutrition research institute federal democratic republic of Ethiopia - Guideline on Measles Surveillance and Outbreak Management, January 2012.

[16] H. Trottier, P. Philippe. Deterministic Modeling of Infectious Diseases: Applications To Measles And Other Similar Infections. The Internet Journal of Infectious Diseases, Volume 2 Number 1, 2001.

[17] Suzzan Lenhart and John T.Workman, "Optimal Control Applied to Biological Models", (2007)

[18] D.J.D. Earn, P. Rohani, B.M. Bolker, B.T. Grenfell, A simple model for complex dynamical transitions in epidemics, Science $287(2000)$

[19] M.J. Ferrari, R.F. Grais, N. Bharti, A.J.K. Conlan, O.N. Bjrnstad, L.J. Wolfson, P.J. Guerin, A. Djibo, B.T. Grenfell, The dynamics of measles in sub-SaharanAfrica, Nature 451 (2008) .

[20] Elsa Hansen and Troy Day, "Optimal control of epidemics with limited resources “, 2000

[21] Ousmane MOUSSA TESSA, Mathematical model for control of measles by vaccination, 2013.

[22] Sofia Rodrigues, M. Teresa T. Monteiro, Delm F. M. Torres, Bio-economic Perspectives to an Optimal Control Dengue Model Helena, January 2013.

[23] Adriana Johnson, Optimal Control of Cell-Cycle-Specific Chemotherapy: An Examination of the Effects of Necrosis, 2010.

[24] S. Nanda, H. Moore, S. Lenhart. Optimal control of treatment in a mathematical model of chronic myelogenous leukemia. Mathematical Biosciences, may 2012.

[25] Federal Democratic Republic of Ethiopia, Population Census Commission. Summary statistical report of the 2007 population and housing census, Addis Ababa: Central Statistics Agency, 2008.

[26] Federal Ministry of Health of Ethiopia, Annual report $2014 / 2015$

[27] Abta Abdelhadi and Laarabi Hassan, Optimal Control Strategy for SEIR with Latent Period and a Saturated Incidence, may 2013.

[28] Abta, Hassan Laarabi, and Hamad Talibi Alaoui, "The Hopf Bifurcation Analysis and Optimal Control of a Delayed SIR Epidemic Model Abdelhadi”, may 2014.

[29] Cristiana J. Silva and Delfim F. M. Torres, Optimal Control of Tuberculosis, 2010

[30] O. Diekmann, P. Heesterbeek, and M. Roberts. The construction of next-generation matrices for compartmental epidemic models. Journal of Royal Society Interface, 2010.

[31] L. Edelstein-Keshet, Mathematical models in biology, 2005

[32] Dorfman, Robert. "An Economic Intepretation of Optimal Control Theory." American Economic Review (December 1969). 

with Optimal Control of Vaccination and Supportive Treatment

[33] Mose Ongau Fred, Johana K. Sigey, Jeconiah A. Okello, James M. Okwoyo \& Giterere J.Kang'ethe, "Mathematical Modeling on the Control of Measles by Vaccination", Vol. 2, No. 3, May 2014
[34] Federal Democratic Republic of Ethiopia, Population Census Commission. Summary statistical report of the 2007 population and housing census, Addis Ababa: Central Statistics Agency, 2008. 